UDC 539

\title{
Models of dark particle interactions with ordinary matter
}

\author{
Takibayev N. \\ Al-Farabi Kazakh National University, IETP, al-Farabi 71, 050040 Almaty, Kazakhstan \\ e-mail:takibayev@gmail.com
}

\begin{abstract}
This paper proposes models of interaction of dark particles with massive bodies. The models describe the resonant amplification of effective interaction between two massive bodies with large distances between them. The phenomenon is explained by the catalytic action of dark particles rescattering in the system of two heavy bodies. We consider the system of two heavy stellar objects where one of them located in a center of a galaxy and another stellar body located at the galaxy's periphery orbiting the center of the galaxy. Resonant amplification of the effective interaction between these two heavy bodies imitates the increase of their mass while their true gravitational mass remains unchanged. Such increased interaction leads to more pronounced gravitational lensing of bypassing light.
\end{abstract}

Key words: dark particle, three-bode systems, resonant amplification of interactions, dark energy.

PACS numbers: 95.35.+d, 03.65.Nk, 21.45.-v

\section{Introduction}

Understanding of the interaction between dark matter particles and usual matter, as well as the nature of dark matter and dark energy, still remains among the challenging problems of modern science. Quite a lot of was collected from observations and analysis of various effects of dark substances such as anomalously high relative velocities of galaxies at high orbits around their common center of mass [1-3] and the phenomenon of gravitational lensing at passing of light through clusters of galaxies [4-6].

The well-known gravitational laws give for the observed total mass in the clusters insufficient values for keeping the galaxies on their peripheral orbits at the observed high velocities. The mass of the clusters of galaxies should be much higher than the observed one. This invisible matter is called as dark matter [1-4]. Moreover, the effect of gravitational lensing has been observed in passing of light through clusters of galaxies, what can also serve as an indication of the presence of dark matter in such clusters [4-7].

Understanding why dark matter is slightly exhibited at smaller distances within galaxies and in small stellar systems is still an enigma; dark matter is almost imperceptible within our solar system [8-13].

Numerical experiments show that dark matter should have existed at early stages of galaxy formation [14-19]. Simulation of processes at those stages (i.e. $\sim 10^{13}$ seconds after the Big Bang) showed that without the dark matter action, the gravitational forces were not able to bind the scattering particles, since their kinetic energies were very high [17-19]. It is the action of dark matter which enabled the formation of atoms, molecules, dust particles and, later, massive objects, stars and galaxies.

The nature of dark matter particles is one of the most intensively discussed issues. There are many theories and ideas about types of dark matter particles, their mutual interactions and interactions with usual matter proposed for solution of related specific and general problems [20-22]. We would like to note that the "dark matter problem" is still unsolved in its core: the nature of dark matter and the character of interaction within dark matter and with particles of usual matter remain undisclosed.

Several particle types, different in their character, are considered to explain the available data. Hypothetical candidates for the dark matter particle are either weakly interacting particles, such as ultralight axions, or massive WIMPs (Weakly Interacting Massive Particles), such as neutralino $[23,24]$. Nevertheless, any small or heavy dark particles have not yet been observed.

The theories like CDM (Cold Dark Matter) and $\Lambda \mathrm{CDM}$ (Lambda Cold Dark Matter) are of particular interest $[20,25]$. They are based on the welldeveloped theoretical approaches. However, these 
theories also faced certain obstacles. Therefore, the galaxy rotation curves due to dark matter action should demonstrate in the CDM the constant density in central parts of the galaxies, while the models for slightly interacting CDM particles give the structures with sharp density peaks the centers of galaxies [26, 27]. Besides that, the calculations provide excessive numbers of CDM clouds in massive halo of the galaxies - for 10 or even 50 times more that the observed ones [20].

$\Lambda \mathrm{CDM}$ is a parameterization of the Big Bang cosmology model with $\Lambda$ as a cosmological constant of the Universe responsible for contributions from dark energy and cold dark matter [20, 26-29]. There are extended $\Lambda \mathrm{CDM}$ models, which take into account cosmology inflation interactions [30], quintessence and other elements studied by cosmology [31-33].

Study of about 400 stars located at distances within 13 thousand light years from our Sun was published in 2012 [12, 16]. It was declared that there is no evidence for noticeable presence of dark matter within this large spacious area while the theories predicted average amount of dark matter in the solar vicinity of about $0.5 \mathrm{~kg}$ per volume equal to the volume of Earth. The measured values gave $0.00 \pm 0.06 \mathrm{~kg}$ of dark matter for this volume. This means that the attempts to register the presence of dark matter on Earth have almost failed [13-15].

According to the Planck space observatory, data interpreted employing the standard cosmological model $\Lambda C D M$ [16-18], the total mass energy of the observed Universe consists of usual (baryon) matter for $4.9 \%$, dark matter for $26.8 \%$ and for $68.3 \%$ of dark energy. Therefore, $95.1 \%$ of the Universe consists of dark energy and dark matter [18].

Discussions of the dark matter problem could be found in thorough reviews and original papers such as [21, 24-26]. General problems related to both dark matter and the dark energy challenges are discussed, for example, in the works [31-34].

\section{The quantum scattering theory for large distances}

In the present paper [35], we discuss specific cases applying the methods of the quantum scattering theory for astrophysical scale systems and considering the interaction of dark matter particles with massive gravitational objects.

The extremely weak interaction of dark matter particles with ordinary matter and enormous
Compton wavelength of dark matter particles make it possible to use the quantum-mechanical approaches in our considerations. Therefore, we take the mass of dark matter particles to be extremely small.

We keep in mind the known quantum features of the particles: Compton wavelength of a particle of mass $m$ determines the range this virtual particle can pass from the place of its origin. Location of a particle can be determined with precision within the Compton wavelength of this particle:

$$
\lambda=h / p=h c / E=h c / m c^{2}=\lambda_{\text {Comp }}
$$

To locate a particle with mass $m$, one needs to irradiate it with photons of the wavelength $\lambda$, which is to be less than the particle localization region; this corresponds to the photon energy $E$.

Photons can generate particles with energy in the localization region $\lambda_{\text {Comp. }}$. A particle cannot be considered as a point object in the localized region $\lambda_{\text {Comp }}$. since part of the time it exists in the state "particle + the pair (particle-antiparticle)". Compton wavelength does therefore determine the minimal uncertainty in the measurement of the particle's coordinate. That is the particle's localization region cannot be determined less than its Compton wavelength.

However, the scale where a particle cannot be considered as a point object depends not only on the particle's Compton wavelength, but also on Compton wavelengths of other particles, which the considered particle can dynamically transform into. For leptons with no strong interaction, for instance, a transition into a different form is a less probable event - one can say it is rare and takes much time. Lepton coat remains therefore transparent and, in many tasks, leptons can be considered as point objects with a high rate of precision.

So, extremely low interaction between a dark particle and ordinary matter can guide us to the following assumptions:

- dark particles are transparent for smallscale objects of ordinary matter (with dimensions $\left.r<<\lambda_{C ; D M}\right)$ and they do not interact with ordinary matter;

- such a dark particle becomes "visible" for small objects of ordinary matter when their scale is $r \geq \lambda_{C ; D M}$, i.e. objects are of the same scale as the dark particle's Compton wavelength; one can consider the interaction potential for a dark particle interaction with such ordinary matter object; 
- dark matter particles do not interact directly with each other at relatively low energies.

Within these assumptions, we can now use in our considerations the quantum-mechanical scattering theory and find possible solutions of this problem. We can use the Lippmann-Schwinger equation solutions [36] for the quantum-mechanical scattering of two bodies with different mass; and the Faddeev's equations for scattering of three bodies with one of the bodies possessing extremely small mass and heavy two other bodies [37,38].

We consider two models for the interaction of dark matter particles with massive gravitational objects; results obtained within these models are discussed, and selective character of dark matter particle interactions with usual matter is explained. Some of the results obtained earlier [35] are incorporated in the present work for the sake of logic and represent ability and for more comprehensive comparison of the newly obtained results with the observed data.

The starting point of the proposed approach is consideration of the main questions: what is the reason for selectivity in the dark matter particle interactions with ordinary matter? More specifically, why and how do the dark matter particles interact more intensively with massive bodies at very large distances, but demonstrate very little or no impact on bodies at relatively small astronomical distances? For instance, why within the solar system is the observed action of dark particles vanishing?

Here we can find an analogy with the catalytic action of an additional third particle on the interaction of two initial particles [35]. The catalytic interactions are known in chemistry [39], and take place in certain cases in the systems of several interacting nuclear particles $[36,38]$.

In our astrophysical three-body models, we consider the gravitational interaction of at least two stellar objects (i.e. two gravitationally interacting bodies), but at the same time, we assume that there is the third sort of objects (dark matter particles). In the sum, this sort of objects introduces an additional interaction between the two massive bodies, and must be considered in the quantum scattering theory of three-body systems [40-42].

In our work, instead of a standard interaction problem with two bodies involved, we consider the problem of several bodies' interaction; more specifically, the three bodies scattering problem. We should note here that in the quantum- mechanical problem of three and more bodies, unique phenomena, that have no analogy in the usual two-body problem, may take place. As examples of such phenomena, one can mention the well-known Thomas effect [43] and the Efimov effect [44],when all three particles that binary interact with each other give anomalous increasing of the number of levels in discrete spectrum. The number of levels is growing as the logarithm of the ratio of the scattering length to the pair force radius [44].

Another example could be the appearance of new resonance states in a system of two heavy particles and a particle with very small mass. In this case, should a pair consisting of one light and one heavy particle has a quasi-stationary state, then new quasi-stationary states appear in a system of three particles at certain distances between two heavy particles [37, 40, 41].

In the next part, we propose two models of interaction between dark particle and heavy body. In first model, the interaction between dark particle and heavy body has a separable form, and a solution for amplitude can be written in the analytical forms. In the second model, the two-body amplitudes are taken in the Breit-Wigner forms.

Then we consider the problem of three bodies' interactions, i.e. the system that consisted of one lightweight particle and two very heavy bodies. While here we deal with the quantum-mechanical problem with a scattering of a lightweight particle in a system of two heavy bodies, we later expand the obtained results for the galactic systems, assuming that the lightweight particle is a dark matter particle and the heavy bodies are galaxies orbiting their center of mass.

We refer to the two heavy stellar objects as a system of one massive body located in a center of a galaxy cluster and another stellar body is the galaxy located at the periphery orbiting the center of the galaxy cluster. These objects interact between each other with gravity forces. Moreover, we consider that the additional forces act between them: it is dark matter interactions [38]. Here, we would like to propose a model for resonance amplification of interaction between two astronomical stellar objects stimulated by additional interaction with dark matter particles (hereinafter-dark particles).

We suppose that the dark particles do not just increase a gravitational mass of stellar objects. More importantly, they noticeably change the effective interaction between these two heavy 
bodies, i.e. two galaxy clusters. The most noticeable factor here is that the orbital velocities at the specific large distances between the heavy bodies become high enough to be registered.

Resonant amplification of the effective interaction between the two heavy bodies imitates an increase in their mass while their true gravitational mass does not change so much. Such increased interaction results in more pronounced gravitational lensing of the bypassing light.

\section{Models of potential for a dark particle with a massive body}

First model. We consider the task in the nonrelativistic case and take into account a simple potential model for interaction of a dark particle with a massive body:

$$
V_{D H}=\left|v_{D H}>g_{D H}<v_{D H}\right|
$$

where $g_{D H}$ is the coupling constant, the subscript $\mathrm{DH}$ denotes an interaction of the Dark particle and the Heavy body. Then, the Lippmann - Schwinger equation takes the form:

$$
T_{D H}=V_{D H}+V_{D H} G_{0}(E) T_{D H}
$$

where $G_{0}(E)$ is the free Green's function of the system. We use the units with $\hbar=1$ and $c=1$.

The solution of (3) $T_{D H}$ also has the following separable form:

where

$$
\begin{aligned}
& T_{D H}=\left|v_{D H}>\eta_{D H}<v_{D H}\right| ; \\
& 1 / \eta_{D H}=1 / \lambda_{D H}+I(E) ;
\end{aligned}
$$

$$
I(E)=-\int \frac{d \vec{p}}{(2 \pi)^{3}} \frac{v_{D H}^{2}(p)}{E-E_{s}+i \cdot 0},
$$

and $v_{D H}=N \frac{t}{1+t^{2}}, t=p / \beta$, where $\beta-$ potential parameter, $\quad N^{2}=4 \pi / m \beta ; \quad E=p_{0}^{2} / 2 m, \quad t_{0}=p_{0} / \beta ;$ $E_{s}=p^{2} / 2 m ; m$ is the mass of a dark particle.

For $S$-wave we obtain:

$$
I(E)=I\left(t_{0}\right)=\frac{1-2 i t_{0}}{\left(1-i t_{0}\right)^{2}} .
$$

Note, $\lambda_{D H}<0$ would correspond to the attractive forces. For $-1<\lambda_{D H}<0$ the amplitude pole corresponds to the virtual level, and for $\lambda_{D H}<-1$ corresponds to the bound state. $\lambda_{D H}$ is real by definition. In the case of $-1<\lambda_{D H}$ there are no bound states for a system of a dark particle and a heavy body.

The value $\eta_{D H}$ is frequently referred to as an amplification coefficient to highlight the similarity between the potential and the $T_{D H}$-matrix forms as well as the dependence of $\eta_{D H}$ on the initial energy of the pair.

Second model. Let us consider the second model for the two-body $T_{B W}$-matrix in Breit-Wigner form assuming that the dark particle has resonant interaction with heavy body of ordinary matter. The $T_{B W}$-matrix can be written in the form:

$$
T_{B W}=\frac{1}{\pi \rho(E)} \frac{\Gamma / 2}{E-E_{R}+i \Gamma / 2}
$$

where $E_{R}$ is the resonance energy and $\Gamma$ is the resonance width, $\rho(E)=m p_{0} / 2 \pi$. We can present this $T$-matrix also in separable form:

$$
T_{B W}=\left|v_{B W}>\eta_{B W}<v_{B W}\right|,
$$

where form-factors can be taken in the simple form

$$
v_{B W}^{2}=1 / \pi \rho(E)
$$

and the resonant part equals

$$
\eta_{B W}=\frac{\Gamma / 2}{E-E_{R}+i \Gamma / 2} .
$$

We suppose that interaction between two dark particles must be extremely small and, therefore, can be omitted. In its turn, the simple model for two-body interaction of a dark particle with a massive body allows to solve the three-body problem analytically.

It is important to note that the two-body interactions between a dark particle and a heavy body themselves cannot increase the interaction between two heavy bodies. However, action of dark matter as catalytic particles can change intensity of interactions between heavy bodies and, remarkably, this influence could be nonlinear with increasing of distances between the heavy bodies.

These solutions distinctly demonstrate the resonance dependence of the effective interaction 
between two massive bodies as a function of distance between these bodies.

So, at relatively small distances the catalytic amplification is negligibly small and effective interaction here coincides with direct (gravitational) interaction between the heavy bodies. At larger distances, the catalytic action of the dark particles increases, and effective interaction of the heavy bodies also increases. At even larger distances, catalytic action can reverse and, instead of attraction between the heavy bodies, cause their repulsion. This to certain extent can emulate the dark energy action [35].

In reality, the nature of interactions between dark particles and a heavy body can be more complex and the catalytic action may happen to be far from simple.

\section{Scattering of a dark particle on a pair of heavy bodies}

Faddeev equations for components of the threebody $T$-matrix can be written as [36]:

$$
T_{i j}(E)=T_{i, D H}+\sum_{l \neq i} T_{i, D H} G_{0}(E) T_{l j}
$$

where $i, j=1,2,3 ; T_{i, D H}$ is the corresponding pare $T$-matrix.

The complete $T$-matrix would correspond to the sum: $T=\sum_{i, j} T_{i j}$. The index $i$ in $T_{i j}$ stands for the number of the pair, which interacts last at the left asymptotic, i.e. corresponds to the number of a body, which first escapes the interaction region. Similarly, the index $j$ denotes the number of the pair, which interacts last at the right asymptotic.

Here we solve the problem taking into account interaction of a dark particle with the one heavy body. The potentials are taken in the separable form (2). At this stage, we assume that there is no pair interaction between the two heavy bodies.

Related components $T_{i j}$ with non-connected and connected parts can be written as:

$$
\begin{gathered}
T_{i j}=T_{i, D H} \delta_{i j}+\mid v_{i, D H}> \\
>\eta_{i, D H} M_{i j} \eta_{j, D H}<v_{j, D H} \mid
\end{gathered}
$$

and transferred into the matrix equations for $M_{i j}$

$$
M_{i j}=\Lambda_{i j}+\sum_{l \neq i} \Lambda_{i l} \eta_{l, D H} M_{l j}
$$

where

$$
\Lambda_{i j}=<v_{i}\left|G_{0}\right| v_{j}>, j \neq i
$$

It is important that the diagonal elements of this matrix are identically equal to zero, i.e. $\Lambda_{i i}=0$. This peculiarity of the Faddeev equations [36] assures compactness of the core of the integral equations.

We consider the case when $m / M \rightarrow 0$, where $m$ is the mass of a dark particle and $M$ is the mass of heavy body. In this limit the total energy of the system is $E_{D M}=p_{0, D M}^{2} / 2 m$, where $p_{0}$ is the initial momentum of the dark particle.

Pair interactions between a dark particle and heavy bodies would not depend on momentums of heavy bodies: $\left\langle v_{2} \mid \vec{p}\right\rangle=v(\vec{p}),\left\langle v_{3} \mid \vec{p}\right\rangle=v(\vec{p})$. Correspondingly, $\eta_{2}=\eta_{3}=\eta\left(p_{0}\right)$ would also be the functions of initial momentum of the dark particle.

The value $\Lambda_{i j}$ takes the form $(j \neq i)[37]$ :

$$
\Lambda_{i j}=2 m v_{i}(\vec{p}) \frac{1}{p_{0}^{2}-p^{2}+i \gamma} v_{j}(\vec{p})=f_{i j}\left(\vec{p}, p_{0}\right) .
$$

We denote with the prime the variables of heavy particles at the reaction outlet; those without a prime - at the reaction entrance. Then $\Lambda_{i j}$ can be written in the form:

$$
\begin{gathered}
\Lambda_{i j}\left(\vec{p}_{i}, \vec{p}_{j}^{\prime}\right)= \\
=\int d \vec{r} \exp \left(i \vec{p}_{i} \vec{r}\right) J_{i j}(\vec{r}) \exp \left(i \vec{p}_{j}^{\prime} \vec{r}\right){ }^{\prime}
\end{gathered}
$$

where

$$
J_{i j}(\vec{r})=\int d \vec{p} \exp (i \vec{p} \vec{r}) f_{i j}\left(\vec{p}, p_{0}\right)
$$

The same transformation for amplitude (9) gives

$$
\begin{gathered}
M_{i j}\left(\vec{r}_{i}, \vec{r}_{j}^{\prime}\right)=\int d \vec{r}^{\prime \prime} \delta\left(\vec{r}_{i}-\vec{r}\right)\left[J_{i j}\left(\vec{r}^{\prime \prime}\right) \delta\left(\vec{r}^{\prime \prime}+\vec{r}_{j}^{\prime}\right)+\right. \\
\left.+\sum_{l=2,3} J_{i l}\left(\vec{r}^{\prime \prime}\right) \eta_{l}\left(p_{0}\right) M_{l j}\left(-\vec{r}^{\prime \prime}, \vec{r}_{j}^{\prime}\right)\right]
\end{gathered}
$$


Since $\delta$-functions call off the integration in the right part of (14), the equation for $M_{i j}\left(\vec{r}_{i}, \vec{r}_{j}^{\prime}\right)$ takes a simple form:

$$
\begin{aligned}
& M_{i j}\left(\vec{r}_{i}, \vec{r}_{j}{ }^{\prime}\right)=J_{i j}\left(\vec{r}_{i}\right) \delta\left(\vec{r}_{i}+\vec{r}_{j}{ }^{\prime}\right)+ \\
& +\sum_{l=2,3} J_{i l}\left(\vec{r}_{i}\right) \eta_{l}\left(p_{0}\right) M_{l j}\left(-\vec{r}_{i}, \vec{r}_{j}{ }^{\prime}\right) \cdot
\end{aligned}
$$

It is remarkable that $\delta$-functions in (19) give the equalities: $\vec{r}_{i}+\vec{r}_{j}{ }^{\prime}=0$ in the case of $j \neq i$, and $\vec{r}_{i}-\vec{r}_{i}{ }^{\prime}=0$. It means that $\vec{r}_{i}$ and $\vec{r}_{j}{ }^{\prime}$ are counted from the center of symmetry between the positions of the heavy bodies. In this case for $\vec{r}_{i}$ and $\vec{r}_{j}{ }^{\prime}$ the indices $i$ and $j$ can be omitted. The solutions can be written in a convenient form [37]:

$$
\begin{gathered}
M\left(\vec{r}, \vec{r}^{\prime}\right)=M^{+}(\vec{r}) \delta\left(\vec{r}+\vec{r}^{\prime}\right)+ \\
+M^{-}(\vec{r}) \delta\left(\vec{r}-\vec{r}^{\prime}\right)
\end{gathered}
$$

where

$$
\begin{gathered}
M^{+}(\vec{r})=\sum_{l}\left[\frac{1}{I-K(\vec{r}) \eta\left(p_{0}\right)}\right]_{i l} J_{l j}(\vec{r}), \\
M^{-}(\vec{r})=\sum_{l}\left[\frac{1}{I-K(\vec{r}) \eta\left(p_{0}\right)}\right]_{i l} K_{l i}(\vec{r}),
\end{gathered}
$$

and

$$
\begin{gathered}
{[K(\vec{r})]_{i j}=K_{i j}(\vec{r})=} \\
=\sum_{l} J_{i l}(\vec{r}) \eta_{l}\left(p_{0}\right) J_{l j}(-\vec{r})
\end{gathered} .
$$

Moreover,

$$
K_{i j}(\vec{r})=0 \text { if } j \neq i,
$$
and

$$
K_{i i}(\vec{r})=\sum_{l} J_{i l}(\vec{r}) \eta_{l}\left(p_{0}\right) J_{l i}(-\vec{r}) \neq 0 \text { if } j=i \text {. }
$$

Note that in our three-body problem we deal with two variables: $p_{0}$ is initial momentum of the dark particle and $r=d / 2, d$ is the distance between two heavy bodies.

\section{Effective interaction between two heavy bodies}

In this section we denote with index $\varphi$ the solutions and interactions in the three body system, but with the $V_{1}$ potential excluded. It means that $V_{\varphi}=V_{2}+V_{3}$ is the sum of potentials corresponding to the interaction of dark particle with each of the two heavy bodies. Then $G_{\varphi}$ is the Green's function with
$V_{\varphi}$ as the potential, and we mark the wave function of this system as $\Psi_{\varphi}$. The solutions are determined in previous section (see eqs. (12) - (23)).

Now one can move forward to solve a problem with inherent pair interaction between heavy particles, taking into account the gravitational potential $V_{l}$. The pair potential $V_{l}$ acts directly between the two heavy bodies of the system. The Lippmann-Schwinger equation for scattering at two potentials can be written as follows $[35,38]$ :

$$
|\Psi>=| \Psi_{\varphi}>+G_{\varphi} V_{1} \mid \Psi>,
$$

where $\Psi(\vec{r})$ is the total wave function for the system that consisted of two heavy bodies, which interact with gravitational force, and one dark particle that interacts with each heavy bodies with the $V_{\varphi}=V_{2}+V_{3}$ potentials. This symbolic equation is a short form of the system of equations in matrix form similar to the Faddeev's equations. The first term in (24) at right describes the resonant behavior, but without direct interactions between two heavy bodies.

Since $G_{\varphi}=G_{0}+G_{0} T_{\varphi} G_{0}$, where $T_{\varphi}$ is the matrix with the components as in (11), we can rewrite (24) in a symbolic form:

$$
\begin{gathered}
|\Psi>=| \Psi_{\phi}>+G_{0}\left(I+T_{\phi} G_{0}\right) V_{1} \mid \Psi>= \\
=\left|\Psi_{\phi}>+G_{0} V_{e f}\right| \Psi>
\end{gathered}
$$

where $V_{e f}=\left(I+T_{\varphi} G_{0}\right) V_{1}, T_{\varphi}=\sum_{i, j} T_{i j}$ with $T_{i j}$ determined in (12). This effective potential corresponds to the distorted potential $V_{l}$ :

$$
\begin{gathered}
V_{e f}=\left(I+T_{\phi} G_{0}\right) V_{1}= \\
V_{1}+\sum_{i, j=2,3}\left|v_{i}>\eta_{i}\left(\delta_{i j}+M_{i j} \eta_{j}\right)<v_{j}\right| G_{0} V_{1} .
\end{gathered}
$$

It then follows that the effective potential as well as the amplitude $M_{i j}(r)$ would depend resonantly on both: the distance between the heavy particles and the energy of the dark particle.

In order to compare the interaction potentials $V_{l}$ and $V_{e f}$ :

$$
V_{e f}=(1+\xi) \cdot V_{1},
$$

acting between the two heavy bodies, it is necessary to record the first potential in the three particle representation. For this purpose, $V_{l}$ can be 
supplemented with Born interaction of the dark particle. A similar addition should be made for the second potential $V_{e f}$. Then the ratio of these quantities leads to the enhancement factor $\xi$ in the case of first potential model:

$$
\xi=I\left(t_{0}\right) \sum_{i, j} \eta_{i}\left(\delta_{i j}+M_{i j} \eta_{j} \cdot F_{j}\right) .
$$

This important outcome demonstrates that the interactions with the third particle can substantially change the character of forces acting between the heavy bodies.

$$
F_{j}=<v_{j} \mid G_{0}=\frac{1}{r \beta}\left[t_{0}\left(\sin \left(k_{0} r\right) c i\left(k_{0} r\right)-\cos \left(k_{0} r\right)\left[s i\left(k_{0} r\right)+\pi / 2\right]\right)-\frac{1}{2 t_{0}}\left(e^{-k_{0} r} \overline{E i}\left(k_{0} r\right)-e^{k_{0} r} E i\left(-k_{0} r\right)\right)\right]
$$

In the case of $\Delta_{i} \rightarrow 0_{+}$, where $1-K_{i i}(\vec{r}) \cdot \eta_{i}=\Delta_{i}$ (see (21), (22)), we obtain

$$
\xi \rightarrow I\left(t_{0}\right) \frac{2}{\Delta} \text {,i.e. }|\xi| \rightarrow \infty \text {. }
$$

The $\xi$ factor in the case of second model will be more complicated. That depends on resonance parameters [38].

\section{Results of calculations}

First model. We introduce dimensionless variables $t_{0}=p_{0} / \beta$ and $\vec{\rho}=\vec{r} \beta$. It is necessary to note that the resonances take place not only when distance between the heavy bodies varies, but also

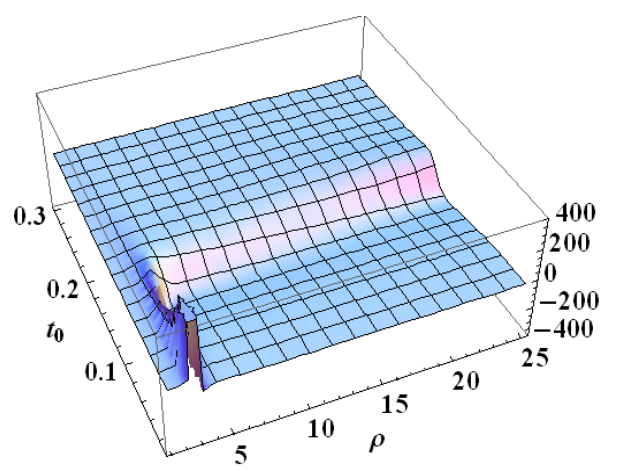

a) $\operatorname{Re}[\xi]$ when the dark particle wavenumber changes. It means that even at resonant distances, the resonance takes place only at certain wave number values of the dark particles. This is a distinguishing feature of resonances in quantum-mechanical of few-body systems as regards to the continuum.

Such phenomenal behavior of the effective interaction between the heavy particles is due to three-particle quantum-mechanical effects. It is important that location of the amplitude pole $M_{i j}^{ \pm}\left(\vec{\rho}, t_{0}\right)$ on the $\vec{\rho}, t_{0}$ plane would change depending on the coupling constant $\lambda_{D H}$ (Fig. 1). But the key factor here is the resonant dependence of the amplitudes $M_{i j}^{ \pm}(\vec{\rho})$ on distance between the heavy particles.

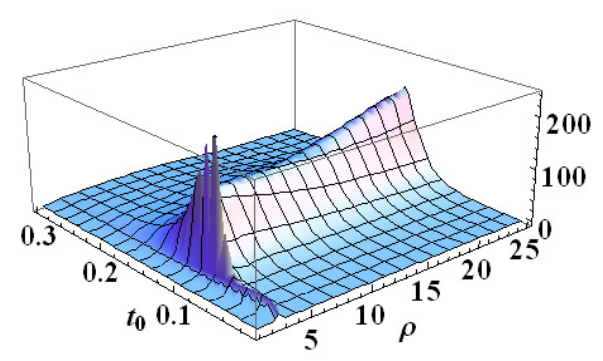

b)- $\operatorname{Im}[\xi]$

Figure $1-$ The behavior of enhancement factor $\xi$ at $\lambda_{\mathrm{DH}}=-0.98$ :

a) $\operatorname{Re}[\xi]$ is the real part of $\xi ; b) \operatorname{Im}[\xi]$ is the imaginary part of $\xi$. 


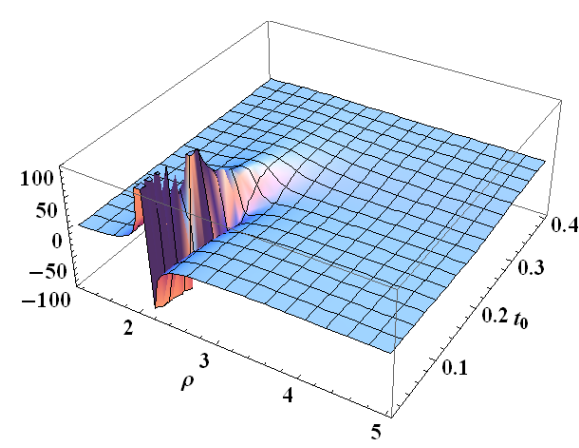

a) $\operatorname{Re}[\xi]$

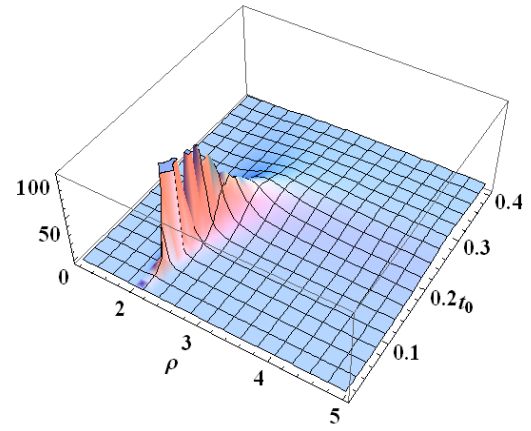

b) $-\operatorname{Im}[\xi]$

Figure 2 - The behavior of enhancement factor $\xi$ at $\lambda_{\mathrm{DH}}=-0.90$ :

a) $\operatorname{Re}[\xi]$ is the real part of ; b) $\operatorname{Im}[\xi]$ is the imaginary parts of $\xi$

Here one should note that the described above model could potentially help in explaining phenomenological interactions at galactic scales. We can now assess the characteristic distances. For example in our model, resonance takes place at the following parameters: $\lambda_{\mathrm{DH}}=-0.98, \rho=r \beta \approx 3$. Assuming that the distance between the massive bodies is about $r \approx 3 / \beta \approx 3 \cdot 10^{22} \mathrm{~cm}$, one can get $\beta \approx 10^{-22} \mathrm{~cm}^{-1}$ and $p_{0} \geq 10^{-23} \mathrm{~cm}^{-1}$. Then, taking into account the resonant behavior of the effective interaction at such distances (see Fig.1), one can get from (27) the following multiplication of the interaction potential for the factor: $\xi \approx 400$ at $\rho \approx 3$.

One can therefore state that the flux of exchange dark particles between heavy gravitating bodies acts like dark matter. The orbital velocity of the peripheral body becomes higher than that at normal gravity owing to the enhancement factor. Moreover, this flux would glue up these two heavy bodies and, with respect to other particles and fields (gamma quanta, for instance) such a system would appear as a single object. The system would have an effective mass much higher than its own mass. It is quite possible that such a mechanism can contribute to gravitational lensing of electromagnetic radiation.

To solve the problem, one can use the FaddeevYakubovski equations [46]. For example, one can follow the symbolic equations (24) - (27) and take into account the additional interaction between electromagnetic wave with ordinary matter. It is quite possible that such mechanism can contribute to gravitational lensing of electromagnetic radiation. Indeed, we can include these additional interactions in our system of equations. Then we obtain new enhancement factor for the electromagnetic waves that gives the results of gravitational lensing.

It is remarkable that the enhancement factors can be negative at certain values of $\rho$ in the case of two-body attractive potentials [38]. For example, in the case of $\lambda_{\mathrm{DH}}=-0.98$ we have $\xi \approx-50-i \cdot 0.1$ at $\rho>3$ and $t_{0}<0.16$ (Fig. 1); in the case of $\lambda_{\mathrm{DH}}=-$ 0.90 we have $\xi \approx-10-i .5$ at $\rho>2.2$ and $t_{0}<0.20$ (Fig. 2). Then the resulting potential between two heavy bodies looks like a repulsive force.

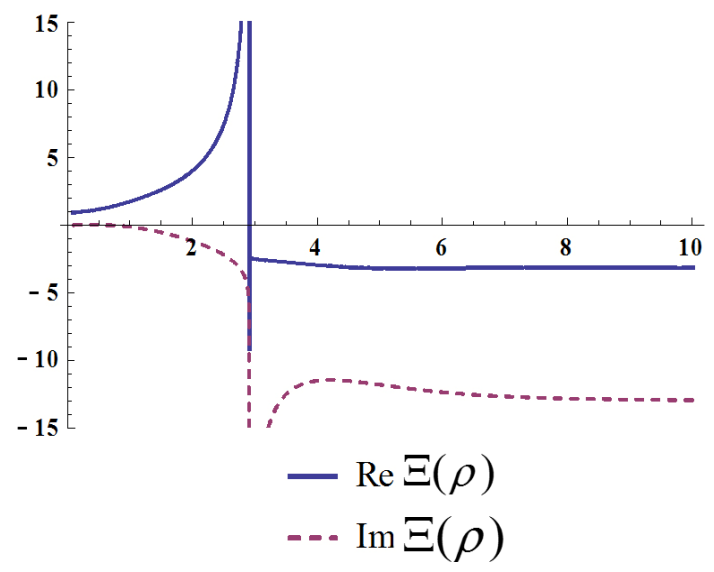

Figure 3 - Real and imaginary parts of $\Xi(\rho) ; \lambda_{\mathrm{DH}}=-0.98$.

The function $\Xi(\rho)$ is the overall result of $\xi\left(t_{0}, \rho\right)$ over the interval $0.001<t_{0}<0.9$. The dependences of the real and imaginary parts of 
$\Xi(\rho)$ at $\lambda_{\mathrm{DH}}=-0.98$ are presented at Fig. 3. We can see that the total action of dark particles gives the attractive force in the region of distances $\rho<3$ and the repulsive one at $\rho>3$. Here the question arises - to what extent can this potential also simulate the action of dark energy?

Second model. In the case of the Breit-Wigner resonances in the systems of a dark particle and a heavy body, such a system would demonstrate a much more complicated picture.

In contrast to the model 1, in the model 2 the resonant amplification occurs in the specific area that is not monotonic over the distance between the heavy bodies. Note, that the resonance enhancement of interactions between the small mass particle and two heavy bodies only happens in some specific cases [37, $40-42]$. Thus, there is the resonance enhancement of interaction between heavy bodies, which can be selective.

In fact, the dark particles with the energies close to resonance energy have to create the intensification of interactions, when the distances between the heavy bodies will correspond to the resonant distances.

The samples of solutions in the case of fourbody problem can be found in [47].
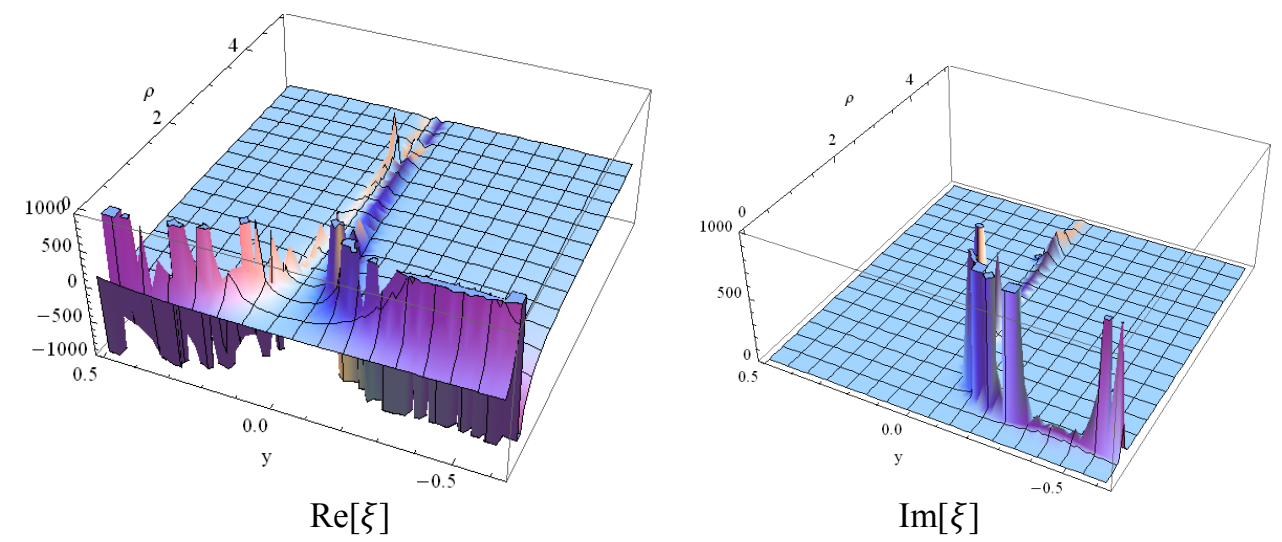

Figure 4 - Real and imaginary parts of $\Xi(\rho)$. The resonance in the system of dark particle and heavy body: $\mathrm{E}_{\mathrm{res}}=1732 ; \Gamma=5$ (conditional units).

a) The case of $\operatorname{Re}[\xi]$; b) the case of $-\operatorname{Im}[\xi]$.
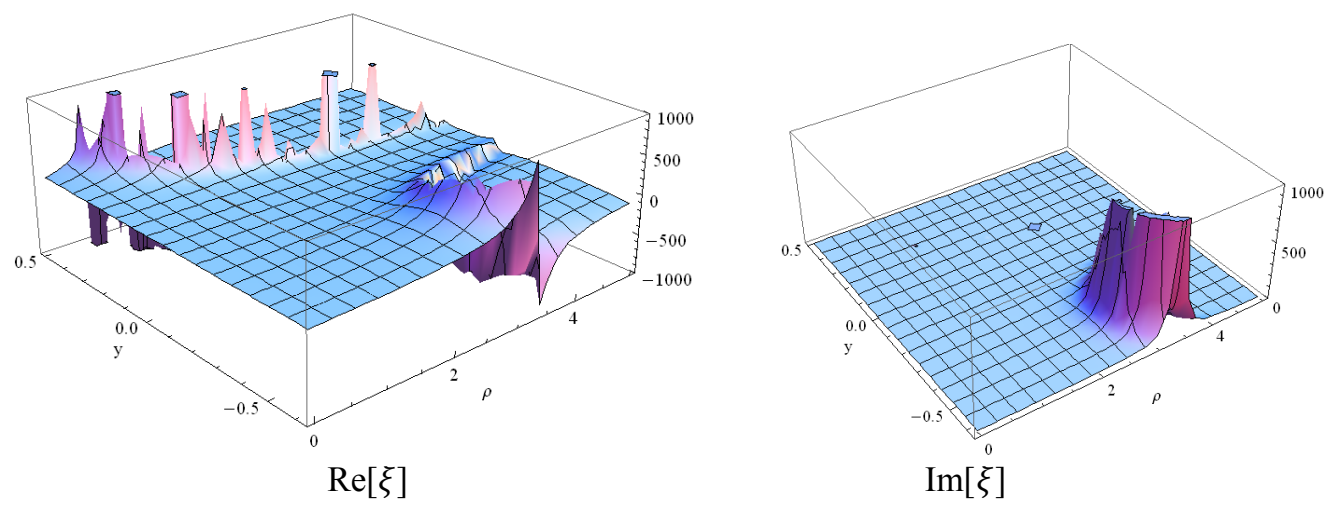

Figure 5 - Real and imaginary parts of $\Xi(\rho)$.

$\Xi(\rho)$ is the enhancement factor in system of a dark particle and two heavy bodies. The resonance in the subsystem of a dark particle and a heavy body has the energy and width: $\mathrm{E}_{\text {res }}=1332 ; \Gamma=35$ (in the conditional units). 
It means that the resonances in the systems of dark matter and two heavy bodies, i.e. two massive objects of astronomical scale in the dark particle clouds would create an amazing structure that cannot be described in the ordinary mechanics.

\section{Discussion}

In this paper we do not determine the nature of dark particles. The problem of dark matter and dark energy is on the current agenda. Here we considered the task of dark particle interactions with ordinary matter within the frame of quantummechanical three-body problems. We tried to understand the co-ordinations between forces that act together in the different subsystems of the threebody system. Then we compared our analytical results with experimental astrophysical evidences. It permits us to ascertain the possible reasons of strong influences to explain some observed data.

For instance, the dark particle coupling constant is taken as $\lambda_{\mathrm{DH}^{3}}>-1$, so the bound states in the pair of the dark particle and the heavy body does not arise. Moreover, we consider the reverse limit when the interaction radius of $\mathrm{DH}$ - forces is taken to be large, but its ratio to the scattering length $\left(1+1 / \lambda_{D H}\right)^{-1}$ remains unchanged.

The direct gravitational interaction between two heavy bodies in the models is complemented by interaction with a third dark particle (or a flow of dark particles, to be more exact). This additional interaction has a specific resonant behavior as a function of the distance between the heavy bodies. The resonant behavior of the effective potential implies its significant increase at certain distances between these bodies.

Figures 1-5 show that the strengthening of effective interaction occurs when repulsion takes place at certain distances. This particular fact reveals a certain similarity in the action of such effective potential and dark matter.

As it follows from the analysis of astronomical observations, dark matter in dwarf galaxies appears to be quite small [11-13], but its effect is huge in big galaxies $[14,15]$. I.e. dark matter interaction is stronger between massive bodies located in the center and on the periphery of a galaxy in a large cluster of galaxies.
Here, we assume that the ensemble of heavy particles in a distant star should be considered as a single massive body, as well as an ensemble of heavy particles in another massive body located in the center of the galaxy. These massive bodies will interact with each other not only via gravity, but also through exchange flows of dark particles.

There is another important fact. Ultra-cold dark particles apparently cannot be registered in terrestrial environments. Indeed, the corresponding wavelengths are large or even of galactic scales. These dark particles do not form bound states with other particles and massive objects. Their impact will be insignificant within the Earth and the Solar System $[11,12]$.

However, the effect of these particles within large galaxies may already be quite noticeable. Strengthening of effective attractive forces will alter the orbits of massive objects in such galaxies.

At even greater distances than the galactic ones, this model provides effective repulsive forces. It requires further investigation to understand how this effect simulates the dark energy action.

\section{Conclusion}

The effect obtained within the proposed model imitates the action of dark matter that leads to amplification of interaction forces at very large but definite distances. An important feature of the resonant amplification is its selectiveness. The effect is negligible at much less distances than the resonance distances. The resonance amplifications are based on well-known quantum-mechanical considerations and, in particular, on the quantum considerations of few-body interactions.

We have considered the gravitational forces in the presence of dark particles, existing in space throughout the universe. This substance can affect the action of normal gravity at very large distances. Although in this paper we do not specify the nature of dark particles and their characteristics, we considered some of the possible effects of these particles on the evolution of our Universe and their resonant interaction.

It should be noted that the interaction of very cold light dark particles with ordinary matter can lead to strengthening of gravitational attraction. 
One should also note that the interaction of light with ordinary matter reinforced in presence of dark particles can result in the lensing effect.

We have noted that the presence of low-energy Breit-Wigner resonances in the interaction of dark particles with ordinary matter may give no monotonic deviations from the trajectories of celestial objects in comparison with the usual effect of gravitational fields.

It also indicates that the interaction of dark particles with ordinary matter at great distances can give its own impact to the effect of dark energy that leads to the accelerating of the dispersal of clusters of galaxies at huge astronomical distances.

\section{Acknowledgement}

The Grant No. 3106 /SF4 from the Ministry of Education and Science of the Republic of Kazakhstan supported this work. The work was carried out at the Institute of Experimental and Theoretical Physics, alFarabi Kazakh National University. The author thanks Dr. Artem N. Yermilov for fruitful discussions and helpful remarks to the object of this paper.

\section{References}

[1] F. Zwicky. On the Masses of Nebulae and of Clusters of Nebulae // The Astrophysical Journal. 1937. - Vol.86. - P. 217.

[2] H. Babcock. "The rotation of the Andromeda Nebula", Lick Observatory bulletin.1939.

[3] W. Ford, Jr. Kent. Rotation of the Andromeda Nebula from a Spectroscopic Survey of Emission Regions // The Astrophysical Journal. - 1970. - Vol.159. - P. 379-403.

[4] XMM-Newton home page.

[5] http://www.cam.ac.uk/research/news/Planck captures portrait of the young Universe, revealing earliest light. University of Cambridge (21 March 2013).

[6] Brooks Michael.13 Things That Don't Make Sense, 2008.

[7] E. Corbelli, P. Salucci. The extended rotation curve and the dark matter halo of M33 // Monthly Notices of the Royal Astronomical Society. - 2000. Vol. 311 (2). - P. 441-447.

[8] D.N. Spergel, P.J. Steinhardt. Observational Evidence for Self-Interacting Cold Dark Matter // Phys. Rev. Lett. - 2000. - Vol. 84. - P. 3760-3763.

[9] Gustavo Yepes, Stefan Gottl, Yehuda Hoffman. Dark Matter in the Local Universe // New Astronomy Reviews. - 2014. - Vol. 58. - P. 1-18.

[10] M.A. Jimenez, X. Hernandez. Local galactic disk stability under MONDian gravity. 2014.arXiv:1406.0537.

[11] C.Moni Bidin. et al. Kinematical and chemical vertical structure of the Galactic thick disk. II. A lack of dark matter in the solar neighborhood // The Astrophysical Journal. - 2012.

[12] Christian Moni Bidin, Giovanni Carraro, ARené. Méndez, Rory Smith, Richard Hook. New study finds mysterious lack of dark matter in Sun's neighbourhood // Science Release, ApJ preprint. - 2012.
[13] G.W. Fraser, A.M. Read, S. Sembay, J.A. Carter, E. Schyns. Potential solar axion signatures in Xray observations with the XMM-Newton observatory // astro-ph.HE, arXiv:1403.2436v2. - 2014.

[14] S. McGaugh. Balance of Dark and Luminous Mass in Rotating Galaxies // Phys. Rev. Lett. 95. 2005.arXiv:astroph/0509305.doi:10.1103/physrevlett.95.171302.

[15] Massimo Meneghetti, Naoki Yoshida, Matthias Bartelmann, Lauro Moscardini, Volker Springel, Giuseppe Tormen, D.M. Simon. Giant cluster arcs as a constraint on the scattering cross-section of dark matter. -2001 , arXiv: astro-ph/0011405v2.

[16] P.A.R. Ade.et al. Planck 2013 results. I. Overview of products and scientific results - Table $9 / /$ Astronomy and Astrophysics.

2013.Bibcode:2013arXiv1303.5062P.

[17] Francis, Matthew. First Planck results: the Universe is still weird and interesting // Arstechnica. - 2013.

[18] Planck captures portrait of the young Universe, revealing earliest light.University of Cambridge, 2013.

[19] T.K. Subramaniam. Dark Matter: An Enigma of Our Universe // AASCIT Communications. - 2015. Vol. 2. - P. 2375-3803.

[20] P. Kroupa, B. Famaey, K.S. de Boer, J. Dabringhausen, M. Pawlowski, C.M. Boily, H. Jerjen, D. Forbes, G. Hensler, M. Metz. Local-Group tests of darkmatter concordance cosmology. Towards a new paradigm for structure formation // A\&A - 2010. - Vol. 523. - P. 32.

[21] P.A. Ade, N. Aghanim, et al. Planck 2015 Results. XIII. Cosmological Parameters. arXiv:1502.01589[astro-ph.CO]. Planck Collaboration, 2015.

[22] Dodelson. Modern cosmology(4. [print.]. ed.). San Diego, CA [etc.]: Academic Press.ISBN9780122191411. -2008 . 
[23] Tiberiu. "Two-fluid dark matter models". Physical Review D83 (12): 124051. arXiv:1106.2642. Bibcode:2011PhRvD..8314051H. H.; Lobo, F. S. N. 2011.

[24] Freese."The Cosmic Cocktail: Three Parts Dark Matter". Princeton, New Jersey: Princeton University Press. ISBN978-0691153353.Katerine. - 2014.

[25] The Dark Matter Group. "An Introduction to Dark Matter". Dark Matter Research (Sheffield, UK: University of Sheffield), 2014.

[26] D.N. Spergel. The dark side of the cosmology: dark matter and dark energy // Science.-2015. - Vol. 347(6226). - P. 1100-1102.

[27] M. Camille. "Planck Upholds Standard Cosmology", Sky \& Telescope, Carlisle, February 10, 2015.

[28] Frieman Joshua, Turner Michael, Huterer Dragan. Dark Energy and the Accelerating Universe // Annual Review of Astronomy and Astrophysics. - 2008. - Vol. 46(1) - P. 385-432.

[29] P.A.R. Ade, N. Aghanim, M. Arnaud. et al. "Planck 2015 Results. XIII. Cosmological Parameters".arXiv:1502.01589[astro-ph.CO]. - 2015.

[30] Andrew Liddle. An Introduction to Modern Cosmology (2nd ed.). - London: Wiley, 2003.

[31] D.N. Spergel. The dark side of the cosmology: dark matter and dark energy // Science. - 2015 - Vol. 347(6226). - P. 1100-1102.

[32] Mattsson Teppo. Dark energy as a mirage // Gen. Rel. Grav. - 2007. - Vol.42 (3). - P. 567-599.

[33] Timothy Clifton, G. Pedro, Ferreira. Does Dark Energy Really Exist? // Scientific American. - 2009. Vol.300 (4). - P. 48-55.

[34] Sean Carroll, Caltech. The Teaching Company, Dark Matter, Dark Energy: The Dark Side of the Universe, Guidebook Part 2 page 46. Retrieved Oct. 7, 2013.
[35] N. Takibayev. Non-relativistic model for interaction of dark particles with matter // Advanced Studies Theor. Phys. - 2014. - Vol. 8. - P. 20.

[36] M.L. Goldberger, K.M. Watson. Collision Theory, Dover Publications, - New York, 2004.

[37] L.D. Faddeev. Mathematical Aspects of the ThreeBody Problem in Quantum Scattering Theory, Davey, - New York, 1965.

[38] N.Zh. Takibayev. Nuclear shell structure in the systematics of nuclear properties // Physics of Atomic Nuclei. - 2008. - Vol. 78. - P. 405.

[39] I. Chorkendorff, J.W. Niemantsverdriet. Concepts of Modern Catalysis and Kinetics, Wiley, ISBN: 978-3-527-31672-4, 477, 2007.

[40] N. Takibayev. Neutron Resonance States in Overdense Crystals // Few-Body System. - 2013. Vol.54. - P. 447-450.

[41] N.Zh. Takibayev. Neutron Resonances in Systems of Few Nuclei and Their Possible Role in Radiation of Overdense Stars // Few-Body Systems. 2011. -Vol. 50. - P. 311-314.

[42] N.Zh. Takibayev. Exact Analytical Solutions in Three-Body Problems and Model of Neutrino Generator // EPJ Web of Conferences. - 2010. - Vol. 3. - P. 05028.

[43] L.H.Thomas. The Interaction Between a Neutron and a Proton and the Structure of $\mathrm{H}^{3} / /$ Phys. Rev. - 1935. - Vol. 47. - P. 903.

[44] V. Efimov. Neutron storage in a magnetic field // Physics of Atomic Nuclei. - 1970. - Vol. 12. - 589.

[45] W. Glockle. The Quantum Mechanical FewBody Problem, Springer, - London, 2011.

[46] O.A. Yakubowsky. A Short introduction to bit string physics // Sov. J. Nucl. Phys. - 1967. - Vol. 5. P. 937.

[47] N.Zh. Takibayev. Solutions of quantum-mechanical problem of light particle on three fixed centers. Izvestiya NAS RK, series phys.-math. $-2010 .-$ N. 3. 